\title{
\begin{tabular}{l|l} 
MitTraries & DSpace@MIT
\end{tabular}
}

\author{
MIT Open Access Articles
}

\section{Self-Organized Translational Wheeling Motion in Stochastic Self-Assembling Modules}

The MIT Faculty has made this article openly available. Please share how this access benefits you. Your story matters.

Citation: Miyashita, Shuhei et al. "Self-Organized Translational Wheeling Motion in Stochastic Self-Assembling Modules." Artificial Life 19.1 (2013): 79-95. (C2013 The MIT Press

As Published: http://dx.doi.org/10.1162/artl_a_00082

Publisher: MIT Press

Persistent URL: http://hdl.handle.net/1721.1/78580

Version: Final published version: final published article, as it appeared in a journal, conference proceedings, or other formally published context

Terms of Use: Article is made available in accordance with the publisher's policy and may be subject to US copyright law. Please refer to the publisher's site for terms of use. 


\section{Self-Organized Translational Wheeling Motion in Stochastic Self-Assembling Modules}

\begin{abstract}
Self-organization is a phenomenon found in biomolecular self-assembly by which proteins are spontaneously driven to assemble and attain various functionalities. This study reports on self-organized behavior in which distributed centimeter-sized modules stochastically aggregate and exhibit a translational wheeling motion. The system consists of two types of centimeter-sized water-floating modules: a triangular-shaped module that is equipped with a vibration motor and a permanent magnet (termed the active module), which can quasirandomly rove around; and circular modules that are equipped with permanent magnets (termed passive modules). In its quasi-random movement in water, the active module picks up passive modules through magnetic attraction. The contacts between the modules induce a torque transfer from the active module to the passive modules. This results in rotational motion of the passive modules. As a consequence of the shape difference between the triangular module and the circular module, the passive modules rotate like wheels, being kept on the same edges as the active module. The motion of the active module is examined, as well as the characteristics and behavior of the self-organization process.
\end{abstract}

\author{
Shuhei Miyashita*,** \\ Massachusetts Institute of \\ Technology \\ Kohei Nakajima ${ }^{\dagger}$
University of Zurich \\ Zoltán Nagy ${ }^{\ddagger}$ \\ ETH Zurich \\ Rolf Pfeifer ${ }^{\dagger}$ \\ University of Zurich
}

Keywords

Self-organized behavior, self-assembly machines, stochasticity, translational wheeling motion, magnetism

A version of this paper with color figures is available online at http://dx.doi.ong/10.1162/ art_a_00082. Subscription required.

\section{Introduction}

Self-assembling robotics is a research field that mainly examines spontaneous manufacturing techniques at unreachable domains. The main aim of self-assembling robotics is to achieve a low-cost and efficient fabrication method, high fault tolerance, and high adaptability, regardless of environmental changes. The approach that this system employs is a so-called bottom-up approach. In this approach, components spontaneously aggregate through local interactions and eventually organize global structures/functionalities. In this way, the systems guarantee high robustness against incidental turbulence and a capability to tolerantly cope with massive parallelism in their aggregation processes. In molecular synthesis, which is arguably the most successful example of self-assembly, some physical conditions are necessary, such

\footnotetext{
* Contact author.

** Distributed Robotics Laboratory, Computer Science and Artificial Intelligence Laboratory, MIT, 32 Vassar St., Cambridge, MA 02139. E-mail: shuheim@csail.mit.edu

$\dagger$ Artificial Intelligence Laboratory, Department of Informatics, University of Zurich, Andreasstrasse 15, 8050 Zurich, Switzerland. E-mail: nakajima@ifi.uzh.ch (K.N.); pfeifer@ifi.uzh.ch (R.P.)

$\ddagger$ Architecture and Sustainable Building Technologies, Department of Architecture, ETH Zurich, Schafmattstr. 32, 8093 Zurich, Switzerland. E-mail: nagy@arch.ethz.ch
} 
as weak interaction, thermal agitation, and nucleation. The mechanisms behind self-assembly are numerous trial-and-error attempts in collisions until the right connection is established. This right connection's strength reaches a sustainable level and hence extends beyond the proofreading bias from the turbulent environment. The system utilizes environmental diffusion as a traveling aid and achieves highly efficient and fast assembly matching. Through such a process, the system gradually shifts to a more energetically stable state. The whole process is fundamentally different from the pick-and-place style, also known as deterministic, of engineering assembly.

In order to provide an overview of the current state and trends of self-assembly research, we investigate related research fields, as well as the approach of this study, in Figure 1. For classification, we took the level of the system's homogeneity or heterogeneity on the $x$ axis, the way modules represent their states on the $y$ axis, and the system's environmental stochastic level on the $z$ axis. Historically, the first advance was made in the field of modular robotics, where robots were made from homogeneous sets of modules and, as a result, featured failure tolerance, such as self-repair capability $[7,23]$. Self-assembly $(\mathrm{SA})$ robotics used a different approach from modular robotics. SA robotics often incorporated stochasticity in its assembly processes, and modules morphed into targeted configurations under that condition. One of the earliest prototypes was proposed by Murata et al. [21]. Their system consisted of a homogeneous set of units, the ability to develop the assembly patterns, and the ability to self-repair the formation. A unique approach realizing amoebic locomotion with many weakly coupled modules can be found in [16]. The system featured resilient body motions, and showed high adaptability to the environmental changes. The introduction of various forms of environmental stochasticity, such as adding mechanical turbulence to the ground [34], air-jet turbulence $[1,10,17]$, and fluid turbulence $[24,32,35]$, has often been tried. Furthermore, the advancement of hardware allows for the possibility of a derivative field that focuses more on practical implementation, such as the approach that is used for medical diagnosis and surgery [25]. A unique approach to self-disassembly using novel electropermanent magnets is presented in [8]. It is important to note that most of the work subscribes to the so-called bottom-up (stochastic) approach. This

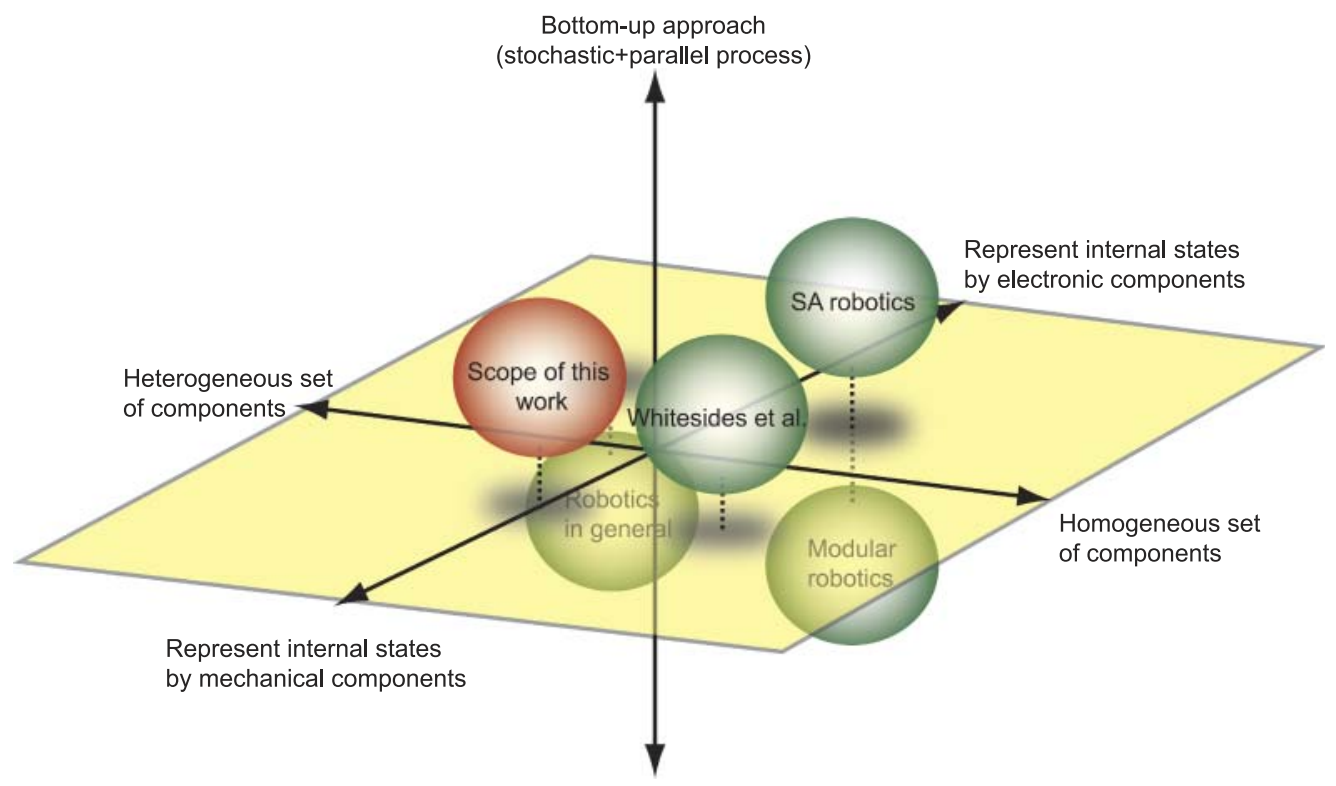

Top-down approach

(deterministic+serial) process

Figure I. Related research fields are described as follows: homogeneity of components ( $x$ axis), module mechanism of internal states ( $y$ axis), and approaches (z axis). 
approach has successfully mimicked biomolecular activities at the macro scale, proving engineered artifacts' self-replication capability, although, due to the frequent use of electronics in the mechanism for internal states, validated capabilities sometimes lack connection to nature.

On the other hand, there is another approach that employs mechanical components for the internal states, and that keeps the environment and assembly process stochastic. An early mechanical model for a self-replicating machine in a stochastic environment was made by Penrose, which simulated natural self-replication [26]. This research was followed by speculation about the clustering patterns of passive elements, focusing on the role of shape in template and component matching [6], as well as on their time evolution [15]. A group led by Whitesides used a chemistryinspired approach, where components feature simple (mechanical/chemical) mechanisms. A series of studies was conducted and showed remarkable achievements in the realization of positional coordinates for molecule-mimetic chemistry [4, 36], circuit functionality [3, 9], reversible aggregation [19], folding structures [2], rotation of magnets [12], and rotation of rotors [11]. Numerous similar research efforts have been devoted to the investigation of morphology [31]. Artificial chemicals that can form in several ways depending on the temperature of the system, such as polymers and dimers, were examined by Breivik et al. [5]. Different aggregation patterns with various sizes of components were shown by Yamaki et al. [37]. An intelligent self-assembling block, which can represent multiple states of the units' rotational angle, was designed by Tsutsumi and Murata [33]. The system can physically express multiple states, conducting exclusive or (XOR) calculation on a $2 \mathrm{D}$ plane.

Morphological computation is a notion that reveals the role of players' morphology in various phenomena, regarding them as computation [28]. The focus can be the elucidation of how a particular body works as computation [13, 27], or developing a design principle for designing a new body for a purpose. Self-assembly has had a close connection to this field, especially when the employed modules show strong mechanical features. For instance, differences in segregation patterns in macroscopic modules are reported in [22].

Having considered the mechanisms of biochemical assembly systems, we derive the prerequisite criteria for our approach as follows.

1. Bottom-up. The system can potentially handle stochasticity and multi-degree parallelism in the assembly processes, where distributed components autonomously assemble without human intervention.

2. Mechanical. To maintain the analogy to biomolecular systems, the module should ideally be mechanically grounded, which also guarantees scalability. The designed mechanism is expected to provide physical causal paths for the assembly processes. Once a set of experimental conditions is invoked, modules are expected to act independently, following local causal rules imposed by the environment.

3. Heterogeneity. Systems that consist of homogeneous components typically offer failure tolerance against physical damage, although such systems generally lack diversity of performance. Our interest in this study is in having different types of components in one environment.

4. Motion by self-organization. In contrast to the high loading capacity of carriage components in modules, accomplished functionalities that can be found in the literature are mainly concerned with structures or simple electric circuits. One of the major advantages of self-assembly systems is the attainment of capabilities that cannot be attained by a single component. We aim to realize a self-organizing motion that meets the abovementioned conditions.

Having considered these four criteria, in this study we focus on attaining translational motion as a self-organized behavior in distributed modules. This kind of motion, which we see in motor proteins in biology, is thought to be crucial in attaining mobilization of self-assembling components. 

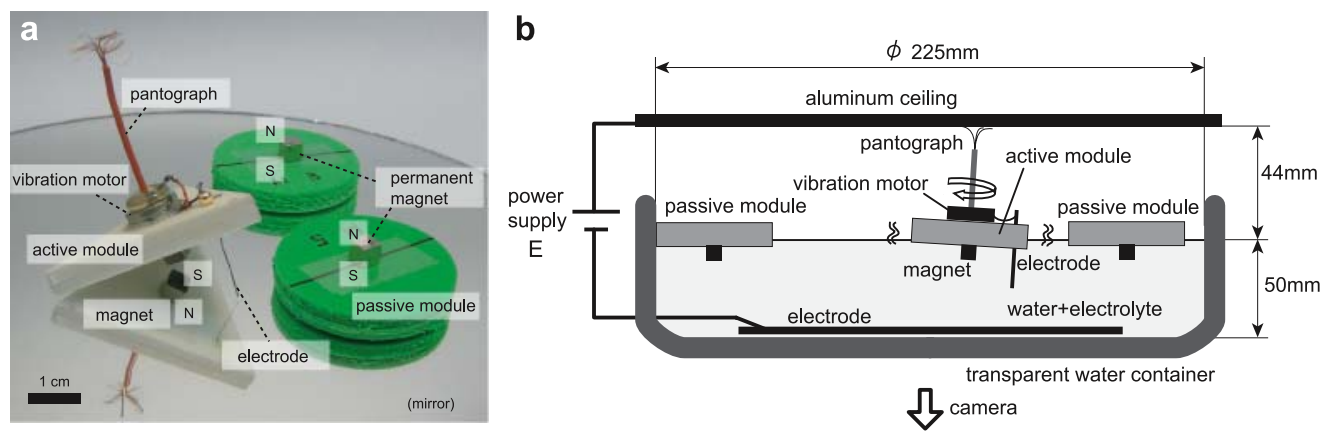

Figure 2. Designed modules and experimental setup. In the electronic version, (a) active module (white triangular module) and passive modules (green circular modules). (b) Scheme of the experiment.

Translational motion not only enables a spatial shift of the components, but also allows them to change the relative position of two connected materials (e.g., kinesins walk on a microtubule and cause muscle contraction).

We present our proposed model, together with an analytical investigation of a single module's motion by simulations, in Section 2. Next we present the attained self-organized behavior in Section 3 . We carefully investigate each phenomenon in Section 4, discuss the results in Section 5, and conclude the study in Section 6.

\section{The Model}

\section{I Experimental Setup}

The experimental platform was designed to enable the systematic investigation of a complex selfassembly phenomenon, which guarantees dissipation and distribution of the system based on [20]. Figure 2 shows (a) the modules and (b) the experimental environment. The modules floated on water. There were two different types of modules: an active module and a passive module. The active module was equipped with a vibration motor on the top of the base plate, which allowed the

Table I. Details of modules and experimental setup.

\begin{tabular}{|c|c|}
\hline Vibration motor & $\begin{array}{l}\text { FM34F, I3,000 rpm }(217 \mathrm{~Hz}) \text {, vibrational } \\
\text { acceleration } 17.6 \mathrm{~m} / \mathrm{s}^{2} \text {, TPC Advanced Technology }\end{array}$ \\
\hline Base plate & Foam rubber, density $1.21 \times 10^{-4} \mathrm{~g} / \mathrm{mm}^{-3}$, Nicole \\
\hline Permanent magnet & $\begin{array}{l}5 \times 5 \times 5 \mathrm{~mm}^{3} \text {, residual magnetism: } 1.3 \mathrm{~T} \text {, } \\
\text { Supermagnete.ch }\end{array}$ \\
\hline Electrode & Platinum, $5 \mathrm{~mm}$ diam $\times 35 \mathrm{~mm}$ \\
\hline Electrolyte (water) & $\mathrm{NaCl}, 83.3 \mathrm{~g} / \mathrm{L}$ \\
\hline Active module & $\begin{array}{l}\text { Edge length } 53.2 \mathrm{~mm} \text {, surface area } 1225 \mathrm{~mm}^{2} \text {, } \\
\text { mass } 3.4 \mathrm{~g}\end{array}$ \\
\hline Passive module & $\begin{array}{l}\text { Diameter } 39.5 \mathrm{~mm} \text {, surface area } 1225 \mathrm{~mm}^{2} \text {, } \\
\text { mass } 1.7 \mathrm{~g}\end{array}$ \\
\hline
\end{tabular}


module to jiggle and move around in its environment, and a permanent magnet at the bottom for attractive or repulsive interactions (see detailed descriptions in Table 1). For the power supply, we opted to supply electricity through a pantograph, which drew current from a metallic ceiling. When a voltage was applied to the ceiling plate, current flowed from the pantograph to the vibration motor and then returned to ground via electrodes that were immersed in the conductive water. During the experiments, an $\mathrm{NaCl}$ solution was used as conductive water. This water enabled sufficient current flow through the chemical reaction $2 \mathrm{NaCl}+2 \mathrm{H}_{2} \mathrm{O} \rightarrow \mathrm{H}_{2} \uparrow+\mathrm{Cl}_{2} \uparrow+2 \mathrm{NaOH}$. Due to this setup, all modules received constant power and were lightweight. Generally, the voltage applied to the ceiling determined the level of perturbation introduced into the system. Thus, it can be regarded as a kind of temperature on a macro scale, which can be used as a regulable parameter. We set up a camera below the water container and observed the modules through the transparent bottom. Table 1 summarizes the components that were installed on each module.

\subsection{Model of Motion of an Active Module}

This section examines the motion of an active module and investigates the influence of a vibration motor. Figure 3 shows a two-dimensional model of the active module's motion, where $\boldsymbol{F}_{\boldsymbol{\omega}}, \boldsymbol{F}_{n}, \boldsymbol{F}_{r}$, $\boldsymbol{F}_{f}, \boldsymbol{F}_{v}, \theta$ are the centripetal force of the eccentric mass in a vibration motor, the frictional force between the ceiling and the pantograph, the resistive force from the ceiling to the pantograph, the buoyancy force acting on the module's body, the drag force of water on the module's body, and the pitch angle of the module, respectively. The values used are listed in Table 2.

Let $\boldsymbol{x}=[x, y, z]^{T}$ be a position vector of the center of mass of the module in a Cartesian coordinate system. Transitional and pitching motions can then be derived from

$M \ddot{\boldsymbol{x}}=\boldsymbol{F}_{\boldsymbol{\omega}}+(M+m) \boldsymbol{g}+\boldsymbol{F}_{f}+\boldsymbol{F}_{r}+\boldsymbol{F}_{n}+\boldsymbol{F}_{v}$,

and

$I \ddot{\theta}=\left\|\boldsymbol{r}_{\omega} \times \boldsymbol{F}_{\omega}\right\|+\left\|\boldsymbol{r}_{\omega} \times m \boldsymbol{g}\right\|+\left\|\boldsymbol{r}_{f} \times \boldsymbol{F}_{f}\right\|+\left\|\boldsymbol{r}_{n} \times \boldsymbol{F}_{r}\right\|+\left\|\boldsymbol{r}_{n} \times \boldsymbol{F}_{n}\right\|$,

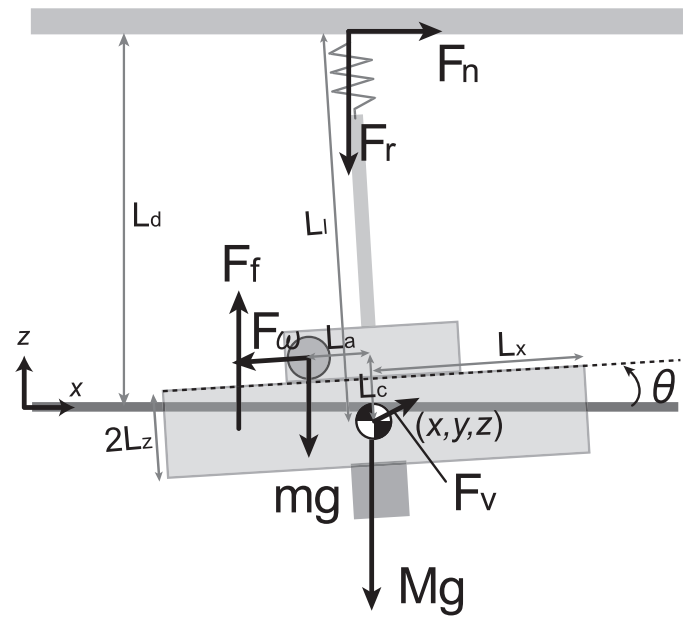

Figure 3. Schematic of a single module. $\boldsymbol{F}_{\omega}, \boldsymbol{F}_{n}, \boldsymbol{F}_{r}, \boldsymbol{F}_{f}, \boldsymbol{F}_{v}, \theta$ are the centripetal force, frictional force, resistive force, buoyancy force, drag force, and pitch angle of the module, respectively. The descriptions of relevant variables are listed in Table 2. 
Table 2. Details of variables. We intentionally used different scales of units (e.g., $g$ and $\mathrm{kg}$ ) for intuitive understanding.

\begin{tabular}{|c|c|c|}
\hline g & Gravitational acceleration vector & $9.81 \mathrm{~m} / \mathrm{s}^{2}$ \\
\hline$m$ & Mass of the eccentric weight & $0.28 \mathrm{~g}$ \\
\hline M & Mass of the module body & $3.12 \mathrm{~g}$ \\
\hline I & Moment of inertia around the center of mass & $9.9 \times 10^{-8} \mathrm{~m}^{4}$ \\
\hline$L_{a}$ & Amplitude of eccentric mass rotation & $5.0 \mathrm{~mm}$ \\
\hline$L_{1}$ & Height of the pantograph & $45.0 \mathrm{~mm}$ \\
\hline $2 L_{x}$ & Side length of the module & $24.4 \mathrm{~mm}$ \\
\hline $2 L_{z}$ & Height of the module & $6.2 \mathrm{~mm}$ \\
\hline$L_{c}$ & $\begin{array}{l}\text { Distance between center of mass and center } \\
\text { of vibration motor }\end{array}$ & $4.8 \mathrm{~mm}$ \\
\hline$L_{d}$ & Distance between the ceiling and water & $45.0 \mathrm{~mm}$ \\
\hline$\omega$ & Angular velocity of vibration motor & $1361 \mathrm{rad} / \mathrm{s}$ \\
\hline k & Spring constant & $39.2 \mathrm{~N} / \mathrm{m}$ \\
\hline c & Drag coefficient & $1.5 \times 10^{-6} \mathrm{~kg} / \mathrm{s}$ \\
\hline$\mu$ & Kinetic friction coefficient & 0.47 \\
\hline$\rho$ & Water density & $1000 \mathrm{~kg} / \mathrm{m}^{3}$ \\
\hline
\end{tabular}

respectively, where $\boldsymbol{r}_{\boldsymbol{\omega}}, \boldsymbol{r}_{f}, \boldsymbol{r}_{n}$, and $\boldsymbol{r}_{v}$ are directional vectors from the center of mass of the module to the action points $\boldsymbol{F}_{\boldsymbol{\omega}}(m \boldsymbol{g}), \boldsymbol{F}_{f}, \boldsymbol{F}_{n}\left(\boldsymbol{F}_{r}\right)$, and $\boldsymbol{F}_{v}$, respectively; and $M, m$, and $I$ are the mass of the module body, mass of the eccentric weight in the vibration motor, and moment of inertia around the center of the module's mass, respectively.

The directional vectors can be represented as

$$
\begin{aligned}
& \boldsymbol{r}_{\omega}=L_{c}[-\sin (\theta), 0, \cos (\theta)]^{T}+L_{a} \cos (\omega t)[-\cos (\theta), 0,-\sin (\theta)]^{T}, \\
& \boldsymbol{r}_{f} \approx\left[\frac{L_{x} \theta}{3 \arctan \left(L_{z} / L_{x}\right)}, 0, \frac{L_{x} \theta^{2}}{3 \arctan \left(L_{z} / L_{x}\right)}\right]^{T}, \\
& \boldsymbol{r}_{n}=\left[-\tan (\theta)\left(L_{d}-z\right), 0, L_{d}-z\right]^{T} \quad\left(\left\|\boldsymbol{r}_{n}\right\| \leq L_{l}\right),
\end{aligned}
$$

where we assume for simplicity that the origin of the buoyant force is on the horizontal centerline of the module, whose distance from the center of mass is proportional to the pitch angle $\theta$. 
Each force can be simplified as

$$
\begin{aligned}
& \boldsymbol{F}_{\omega}=m \omega^{2} L_{a}[\cos (\omega t) \cos (\theta), \sin (\omega t), \cos (\omega t) \sin (\theta)]^{T}, \\
& \boldsymbol{F}_{f} \approx\left[0,0,2 \rho g L_{x}^{2}\left(-z+L_{z} \cos (\theta)\right)\right]^{T}, \\
& \boldsymbol{F}_{r}= \begin{cases}{[0,0,0]^{T}} & \left(\left\|\boldsymbol{r}_{n}\right\|>L_{l}\right), \\
-k\left(L_{l}-\left\|r_{n}\right\|\right)[0,0,1]^{T} & \left(\left\|\boldsymbol{r}_{n}\right\| \leq L_{l}\right),\end{cases} \\
& \boldsymbol{F}_{n}= \begin{cases}{[0,0,0]^{T}} & \left(\left\|\boldsymbol{r}_{n}\right\|>L_{l}\right), \\
\operatorname{sign}\left(\boldsymbol{r}_{n}\right) \mu\left\|\boldsymbol{F}_{r}\right\|[1,0,0]^{T} & \left(\left\|\boldsymbol{r}_{n}\right\| \leq L_{l}\right),\end{cases} \\
& \boldsymbol{F}_{v} \approx-c[\dot{x}, \dot{y}, \dot{z}]^{T},
\end{aligned}
$$

where $k$ is the spring constant of the pantograph, $\mu$ is the kinetic friction coefficient, and $c$ is the coefficient of the water's drag force. Note that the flickering contact of the pantograph with the ceiling caused by the vibration added some fluctuations to the current flow through the motors. However, since the drive of the motor is mainly affected by the supplied power, which can be temporally averaged, we believe that the influence of that perturbation is negligible and do not incorporate it into the model. Note that the rotational speed of the eccentric mass is roughly proportional to the voltage applied. As this speed increased, the modules moved faster and there were stronger collisions between them.

Figure 4 illustrates the numerically obtained simulation results for the motion of an active module. In the simulation, initial values were set as $\left[x, x^{\prime}, z, z^{\prime}, \theta, \theta^{\prime}\right]=0$. We compare the results by varying the rotational frequency of the vibration motor: $12,000,13,000$, and 14,000 rpm, which roughly correspond to $5.5,6.0$, and $6.5 \mathrm{~V}$ of voltage applied to the ceiling. These values are derived by referring to the plot in the data sheet for the product.

Figure 4 a shows the time evolution of the $x$ and $z$ positions of a module. In addition to the highfrequency oscillations seen in the $x$ direction, which are caused by the vibrational motion, long periods of oscillations at about $10 \mathrm{~Hz}$ in the z direction can be recognized in each case. Moreover, the magnitudes of this low-frequency oscillation along the $₹$ direction depend on the rotation speeds of the vibration motors. Translational motion along the $x$ direction, especially over a longer period

a

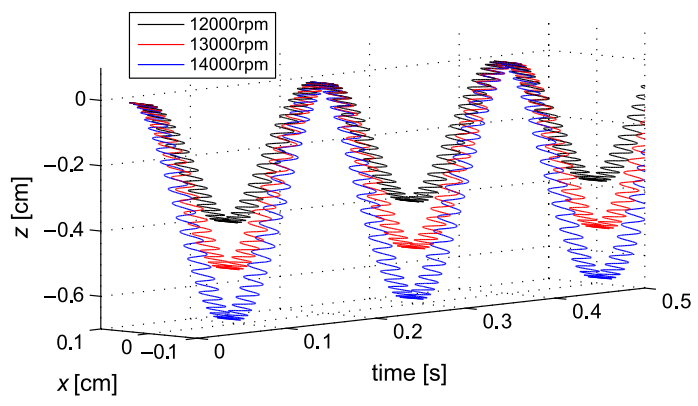

b

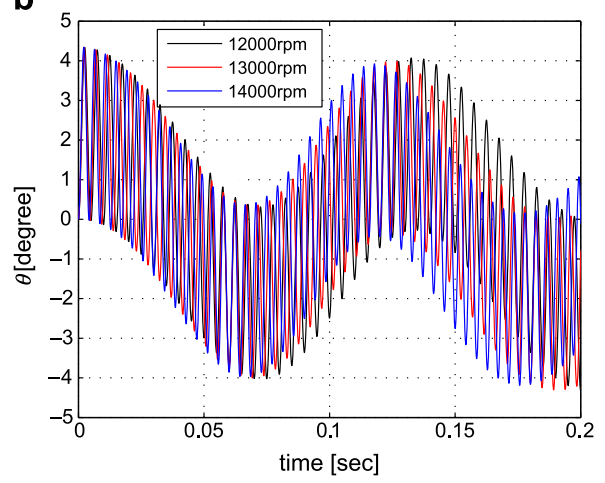

Figure 4. Simulation results for motion of an active module. (a) Time evolution of $x$ and $z$ motion. (b) time evolution of pitch angle of the module. We set the rotational frequency of the vibration motor at $12,000,13,000$, and I4,000 rpm, which approximately corresponded to $5.5,6.0$, and $6.5 \mathrm{~V}$ of applied voltage to the ceiling. 
(e.g., 10 s), was observed in each case. However, these displacements were usually small (e.g., not more than $1.5 \mathrm{~cm}$ in $10 \mathrm{~s}$ ) compared to the experimentally obtained data and were not easily distinguishable from the accumulated numerical errors, which are not discussed in this research.

Figure $4 \mathrm{~b}$ shows the time evolution of the pitch angle of a module $(\theta)$. Note that the maximum angle in practice is $\pm 14.25^{\circ}$ ( $\pm 0.249 \mathrm{rad}$ ), which occured when an edge of the bottom surface of the module was about to be detached from the water surface, or the top surface of the module was about to go below the water surface. The simulation result showed two different frequency scales. In addition to the approximately $200-\mathrm{Hz}$ high-frequency wave caused by the vibrational motion, the module tilted alternately in opposite directions approximately every $0.13 \mathrm{~s}$. Faster vibration caused an increase not only in the high-frequency oscillation, but also in the low-frequency oscillation.

\subsection{Magnetic Interaction}

The magnets on the modules provided long-range interaction between the modules as follows. Consider two magnetic dipoles $\boldsymbol{m}_{i}$ and $\boldsymbol{m}_{j}(i, j \in \mathbb{N}, i \neq j)$ at the centers of mass of modules $i$ and $j$ separated by a distance $r_{j i} \gg d(d=$ side length of the magnet). The magnetic flux density at the position of $\boldsymbol{m}_{i}$ created by $\boldsymbol{m}_{j}$ can be described with a position vector $\boldsymbol{r}_{j i}$ as

$\boldsymbol{B}_{i j}=\frac{\mu_{0}}{4 \pi}\left[\frac{3\left(\boldsymbol{m}_{j} \cdot \boldsymbol{r}_{j i}\right) \boldsymbol{r}_{j i}}{r_{j i}^{5}}-\frac{\boldsymbol{m}_{j}}{r_{j i}^{3}}\right]$,

where $\mu_{0}=4 \pi \times 10^{-7} \mathrm{~T} \mathrm{~m} / \mathrm{A}$ is the permeability of free space.

The magnetic force, torque, and potential energy acting on $\boldsymbol{m}_{1}$ due to $\boldsymbol{m}_{2}$ are respectively given as

$$
\begin{aligned}
\boldsymbol{F}_{i j} & =\left(\boldsymbol{m}_{i} \cdot \nabla\right) \boldsymbol{B}_{i j}, \\
\boldsymbol{\tau}_{i j} & =\boldsymbol{m}_{i} \times \boldsymbol{B}_{i j}, \\
U_{i j} & =-\boldsymbol{m}_{i} \cdot \boldsymbol{B}_{i j} .
\end{aligned}
$$

Since the magnets were mounted onto the modules along the $z$ axis, we consider them as either parallel or antiparallel for the interaction. Then Equations 12-14 are simplified as follows:

$$
\begin{aligned}
\boldsymbol{F}_{i j} & =\sigma_{i j} \frac{3 \mu_{0}}{4 \pi} \frac{m_{i} m_{j}}{r_{i j}^{5}} r_{j i}, \quad \sigma_{i j}=\frac{\boldsymbol{m}_{i} \cdot \boldsymbol{m}_{j}}{\left|\boldsymbol{m}_{i}\right|\left|\boldsymbol{m}_{j}\right|}, \\
\boldsymbol{\tau}_{i j} & =\mathbf{0}, \\
U_{i j} & =\sigma_{i j} \frac{\mu_{0}}{4 \pi} \frac{m_{i} m_{j}}{r_{i j}^{3}}, \quad \sigma_{i j}=\frac{\boldsymbol{m}_{i} \cdot \boldsymbol{m}_{j}}{\left|\boldsymbol{m}_{i}\right|\left|\boldsymbol{m}_{j}\right|} .
\end{aligned}
$$

These expressions imply that the attraction and repulsion of the two magnets were determined by their orientations and that the magnetic force simply decayed in inverse proportion to the fourth power of the distance $\left(\propto r^{-4}\right)$. The torque acting on a single module is zero. This is one of the advantages of the system, since, when the interactions of modules are further developed, we only need to consider the distances. 
We can determine the total potential energy of the system as

$U_{\text {total }}=\sum_{i>j} U_{i j}$

Finally, we normalized the energy as $U_{\text {total }}^{\prime} \equiv U_{\text {total }} /\left(\frac{\mu_{0}}{4 \pi} m^{2}\right)$, assuming that all of the magnets are equally magnetized as $\left(m_{i}=m_{j}=m \forall i, j\right)$. The system minimizes the potential magnetic energy over time. The value and the shape of the function represent the preferable configuration and the stability of a system.

\section{Self-Organizing Translational Wheeling Behavior}

Figure $5 a-i$ show behavior that was typically observed in the experiments. As an initial condition, we manually placed an active module at the center of the container and positioned three passive modules (colored green) along the wall so that they were equally distributed (Figure 5a). Once a voltage was applied, the following actions took place: translational motion of the active module, contact with the passive module (the first assembly), and rotation of the passive module about an edge of the active module (as soon as an assembly occurs, the circular module begins to rotate: Figure 5b), wallfollowing behavior driven by the active triangle module (Figure $5 \mathrm{c}, \mathrm{d}$ ), the second and the third assembly (Figure 5c and Figure 5e, respectively), rotational movement (Figure 5f, g), and wall following (Figure $5 \mathrm{~h}, \mathrm{i}$ ). The rotational movements were observed when at least one passive module was attached to an active module and the whole body did not have any contact with the wall.

The assembly motion, the rotational movement, and the wall-following behaviors can also be recognized in Figure 5j, where the time evolution of the $x$ positions of all the modules is displayed. The rotation of the whole body can be recognized as $120^{\circ}$ phase delay (highlighted in dotted boxes), and the wall-following behavior can be seen as coherent phases (also highlighted in dotted boxes). These dynamical motions are thought to be attractors when there is no influence from the wall.

The transition of the system's magnetic potential energy is shown in Figure 5k. The energy drops can be identified when assemblies occur. It is shown that the energy is mainly consumed for the assembly and has little to do with the motion. Compared to the rotational phase, the wall-following phase shows periodic rises and falls in energy transition. This is because the active module often changes its relative position to the two wheeling passive modules: The active module occasionally sank into the passive modules. Nevertheless, the system maintains a stable translational motion, as can be seen in Figure 5j, proving the intrinsic robustness of the model.

In the following section, we investigate different observed behaviors classifying into assembly process (Section 4.1), rotational movement (Section 4.2), and wall following (Section 4.3).

\section{Investigations}

\section{I Self-Assembly Process}

Assembly was mainly managed by magnetic attraction, though it has little influence when modules are apart. An active module has to rove around the field and have physical contact with a passive module. We have plotted two examples of the trajectory in Figure 6, where an active module picked up two passive modules (the third assembly normally takes a long time and is, as a result, excluded from the plot). The trajectories clearly show distinct correlated movements of passive modules when assembly occurs. This shows that the influence of magnetic attractions is mainly effective when two modules are in contact.

Figure $7 \mathrm{a}$ shows the time that was needed for the first and the second assembly. Note that we define the trials whose assembly interval (first to second) took more than $30 \mathrm{~s}$ as trapped trials, and they were eliminated from the statistics. Such trials were observed in 6 out of the 21 trials (success 

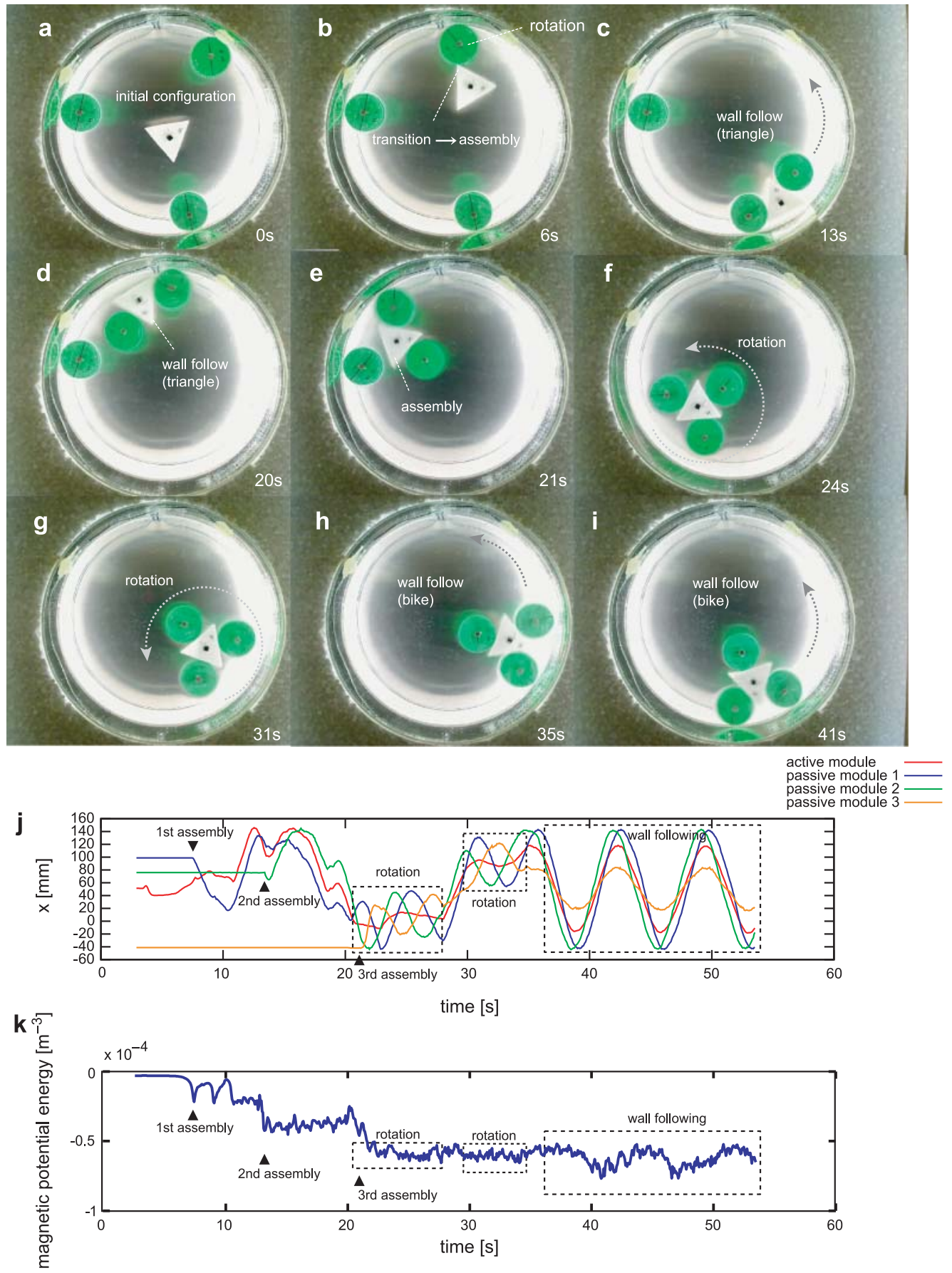

Figure 5. Representative experimental run, consisting of assembly motion (initial condition, a; Ist assembly, b), wall following driven by an active triangle module (c, d), third assembly (e), rotational movement (f, g), and wall following indirectly managed by passive modules $(\mathrm{h}, \mathrm{i})$. Time evolution of $x$ positions of all the modules $(\mathrm{j})$ and normalized magnetic potential energy $(k)$. 

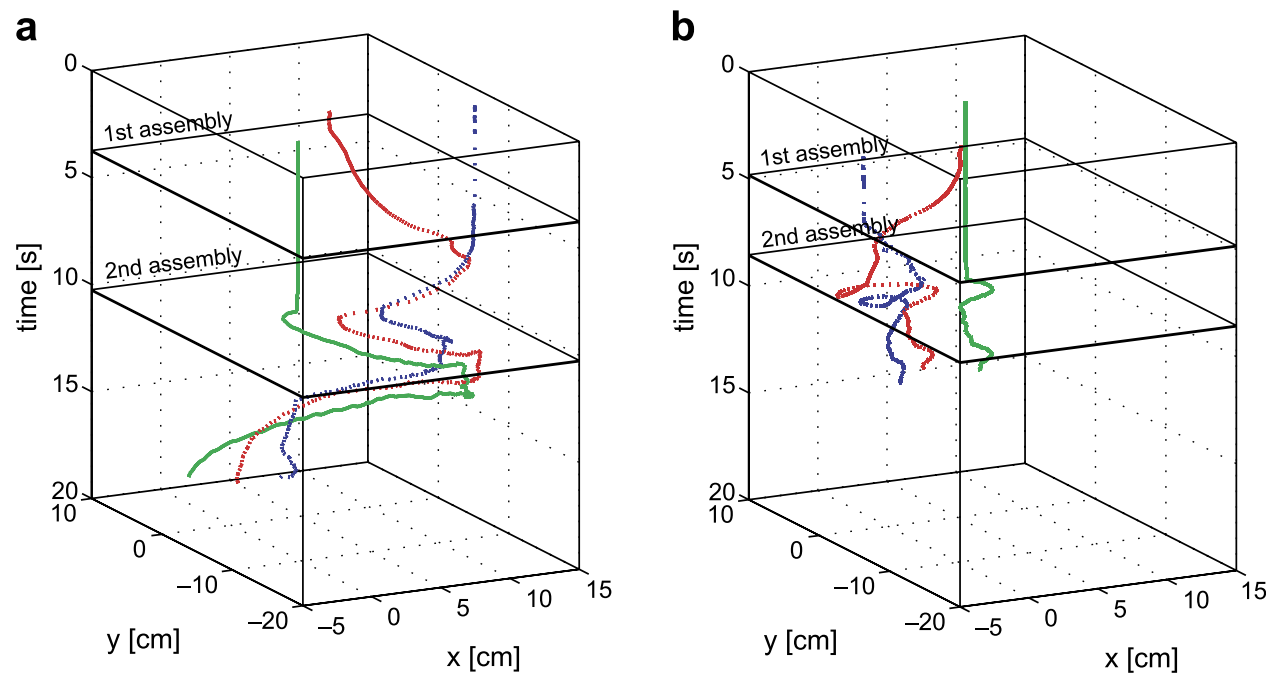

Figure 6. Assembly process (trajectory). a and b are different randomly chosen trials; one can see the difference in time taken and correlated motions after assembly.

rate $=71.4 \%)$. The average time of the first assembly was $2.92 \mathrm{~s}( \pm 2.53 \mathrm{~s})$, and the average time of the second was $11.88 \mathrm{~s}( \pm 6.38 \mathrm{~s})$.

It is worth noting that the second assembly took longer in general, and the variance of the times was high. The reason for this is that the magnetic fields canceled each other out, making other magnetic interactions weaker than in a single module-module interaction. See also Figure 8 and its discussion below. We observe typical trapped states in Figure 7b, c. An active (vibration-motorequipped) triangle and a passive circle driven by the active module pulled each other in opposite directions and consequently stayed in the same place (Figure 7b). This happened because there is only one active module. As a result, convergence to the local minimum state should be avoided if there is another active module. Also, an observed different locally stable configuration is illustrated in Figure $7 \mathrm{c}$, where a carried passive module pushed other passive modules and was prevented from connecting to the active module.

a

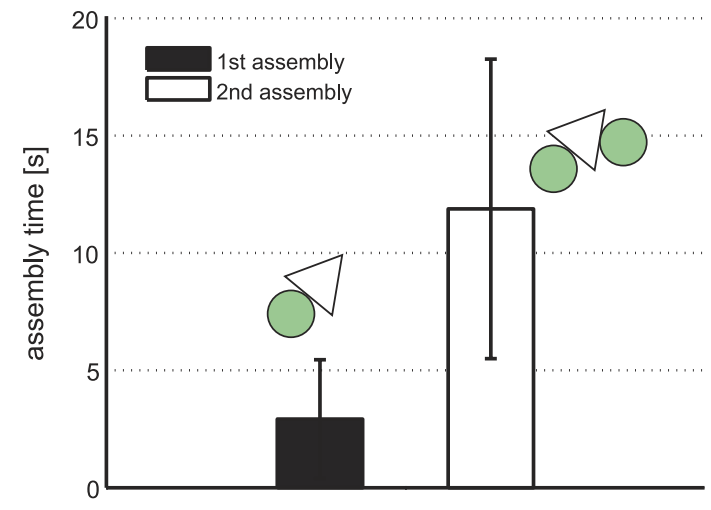

b
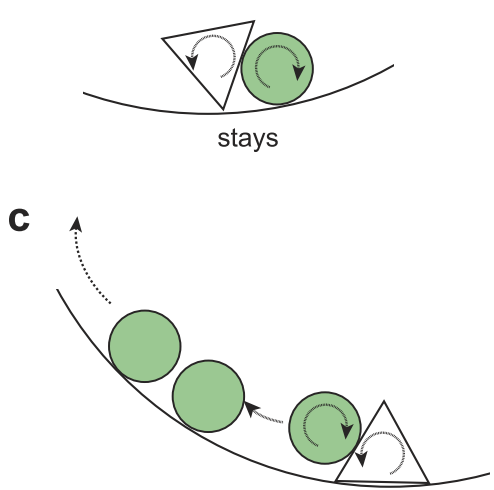

travels, but pushes away

Figure 7. Assembly time (7.0 V, $n=15)$. (a) Assembly time taken for first and second assembly. (b, c) Typical trapped situations. 

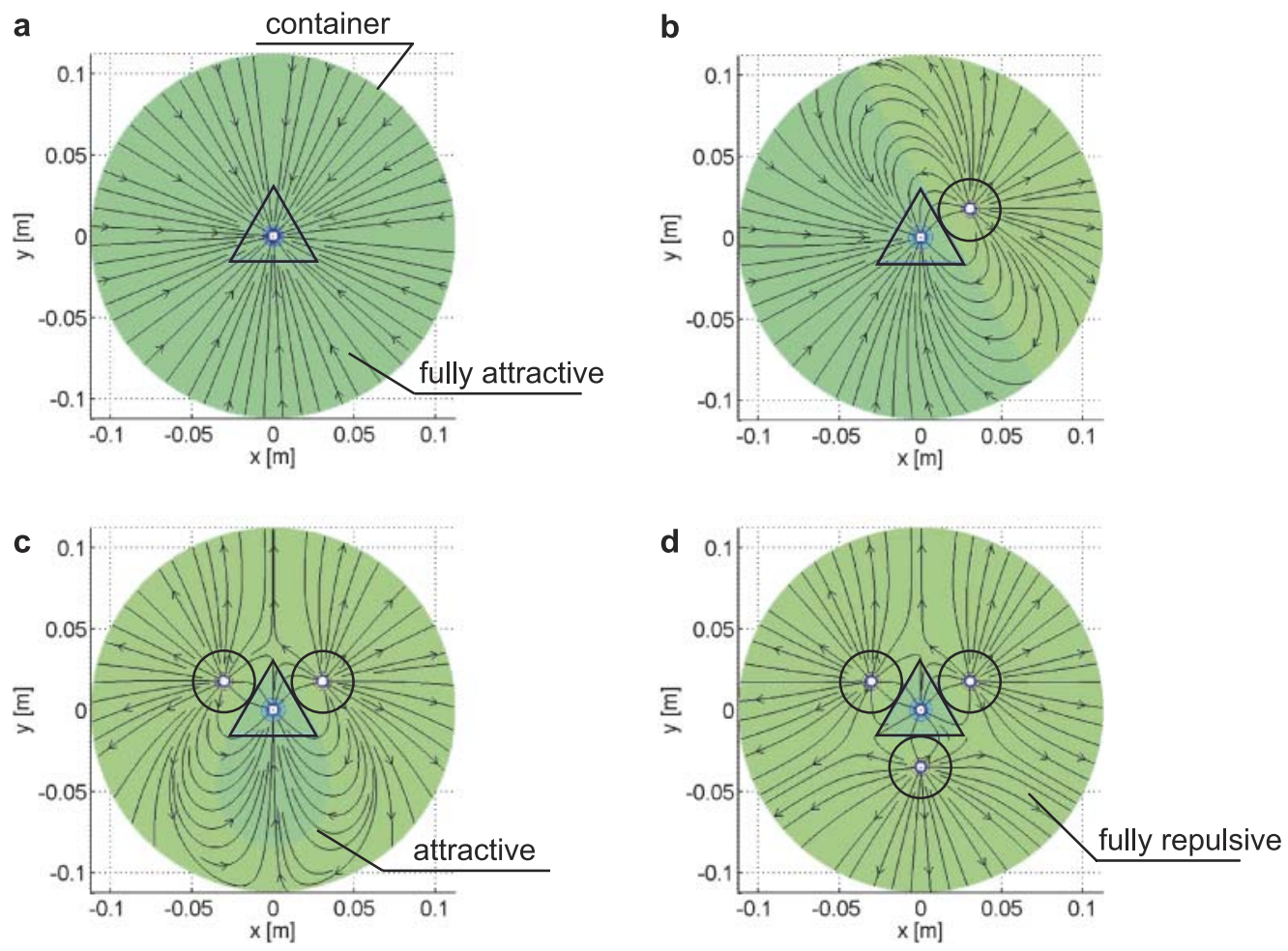

Figure 8. Attractive regions at different assembly stages: Only the active module exists in the container (a); one passive module is attached to the active module (b); two passive modules are attached (c); three passive modules are attached (d).

In order to see the influence of the first assembly, we visualized terrains of magnetic potential energy of different assembly stages in Figure 8. We place modules as displayed: Only the active module exists in the container (a), one passive module is attached to the active module (b), two passive modules are attached (c), and three passive modules are attached (d). The streamlines are derived from Equation 18, representing the gradient decay of $U_{\text {total }}$. Namely, they show the paths of an additional passive module in the field. It can be seen that, as the assembly process proceeds from Figure $8 \mathrm{a}$ to Figure $8 \mathrm{~d}$, the attractive region of the active module drastically decreases. In Figure 8a, a passive module is attracted omnidirectionally; in Figure 8b, due mainly to the boundary effect, the attractive region shrinks almost to half the size (ideally a second passive module should reach the active module following the path); in Figure 8c, the attachment of the second passive tile further decreases the attractive region; and in Figure 8d, the complete assembly does not provide a significant attractive region. The comparison between Figure $8 \mathrm{a}$ and Figure $8 \mathrm{~d}$ tells us that the force is omnidirectionally attractive in Figure $8 \mathrm{a}$ and it is omnidirectionally repulsive in Figure 8d. That means that if another group existed in the field like the one in Figure 8d, then they would repel each other.

\subsection{Rotation of a Group}

A rotational motion was observed when at least one passive module had a connection to an active module, while none of the modules was touching the container boundary. Figure $9 \mathrm{a}, \mathrm{b}$ shows the experimentally obtained time evolutions of the rotational motions' trajectories. The rotational velocities were $1.14 \mathrm{rad} / \mathrm{s}$ in Figure $9 \mathrm{a}$, and $0.70 \mathrm{rad} / \mathrm{s}$ in Figure $9 \mathrm{~b}$. The directions of rotation were the same all the time and determined by the rotational direction of the vibration motor. The rotational speeds vary, especially in the case of Figure 9b. Being hindered in its motion, the passive module 
a

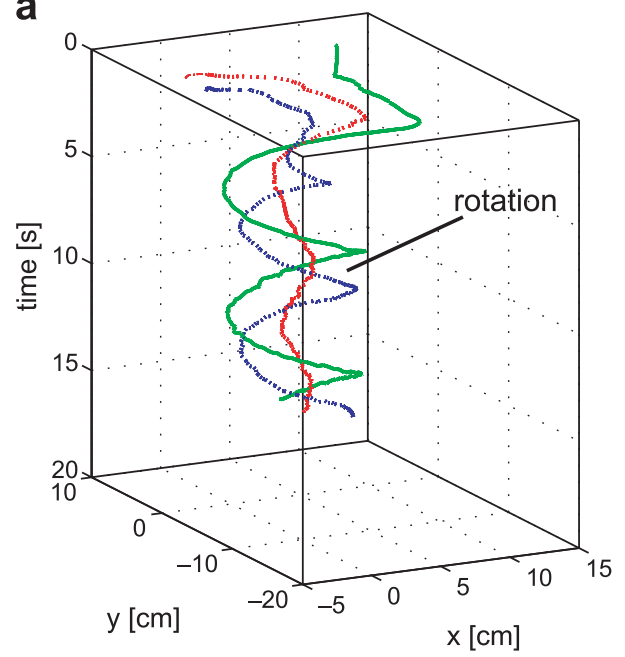

b

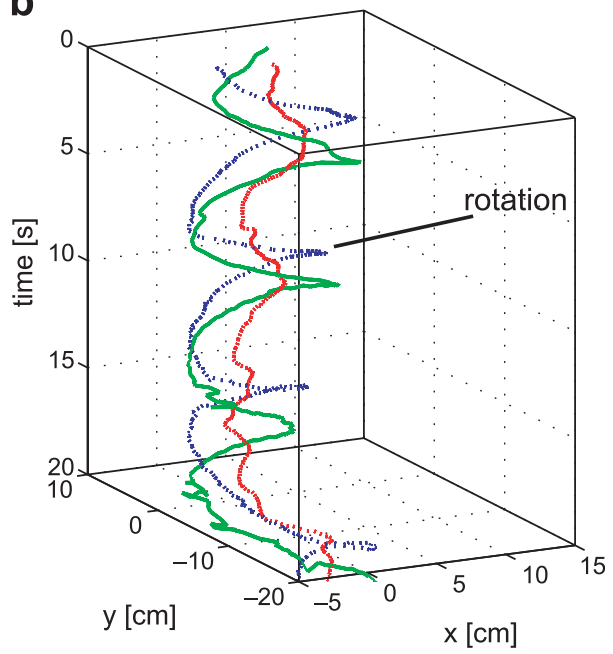

Figure 9. Rotational motion. (a) and (b) are from different trials. The rotational behavior is stably observed, and it usually lasts until the group hits the wall of the container.

occasionally contacts the boundary. The contact times vary, due to the relative positions of the whole group to the boundary, while the motion was stable.

Rotation of an assembled group (at least one passive and one active module) follows from the conservation of the angular momentum, since no external torque is applied to the cluster. The frictional collisions between the modules induce a friction force, which in turn generates a torque in both modules in the same direction, leading to a nonzero total torque on the cluster. This torque must be balanced to fulfill the conservation of the angular momentum and thus yield the observed rotation of the cluster.

\subsection{Wall Following and Morphology Dependence}

Wall-following behaviors were frequently observed in the experiments, though they did not necessarily occur when passive modules acted as wheels. Such behavior was caused either by an active triangle module (Figure 10b) or indirectly through two passive modules (Figure 10c). The requirement for this phenomenon is a large capillary force between the wall and the modules having contact with the wall. We roughly estimate the capillary force using magnets, measuring the distance at which a magnet causes detachment of a module placed on a wall of the container. We use the same strength of magnets as listed in Table 2 and derive the average distance of $3.73 \mathrm{~cm}$ with a standard deviation of $0.25 \mathrm{~cm}$. Taking the magnetization into account, we obtain $0.26 \mathrm{~N}$ as the average capillary force between a module and the container.

We examined the speed of the wall following for different applied voltages shown in Figure 10a. In general, increases in velocity were produced by increases in applied voltage in both cases (4.67 to $10.07 \mathrm{~cm} / \mathrm{s}$ with triangle, and 7.68 to $10.22 \mathrm{~cm} / \mathrm{s}$ with bike). However, the speeds of the triangle and bike configurations at $7 \mathrm{~V}$ were not significantly different. Note that the velocities can be changed to negative by applying negative voltage to the system.

We further examined the effect of morphology by magnifying the size of passive modules (Figure 10d). Interestingly, the speed was smaller in both conditions, 5.5 and $7.0 \mathrm{~V}$, than with normal passive modules $(18.1 \%$ decrease at $5.5 \mathrm{~V}$, and $12.9 \%$ decrease at $7.0 \mathrm{~V}$, from the normal bike configuration). This suggests that there exists an appropriate size match between active and passive modules.

\section{Discussions}

\section{I Reliability Under Environmental Changes}

The reliability of the presented system under environmental changes (the size of the container, the applied voltage, or the viscosity of the water) is an important question. These conditions have not 
a

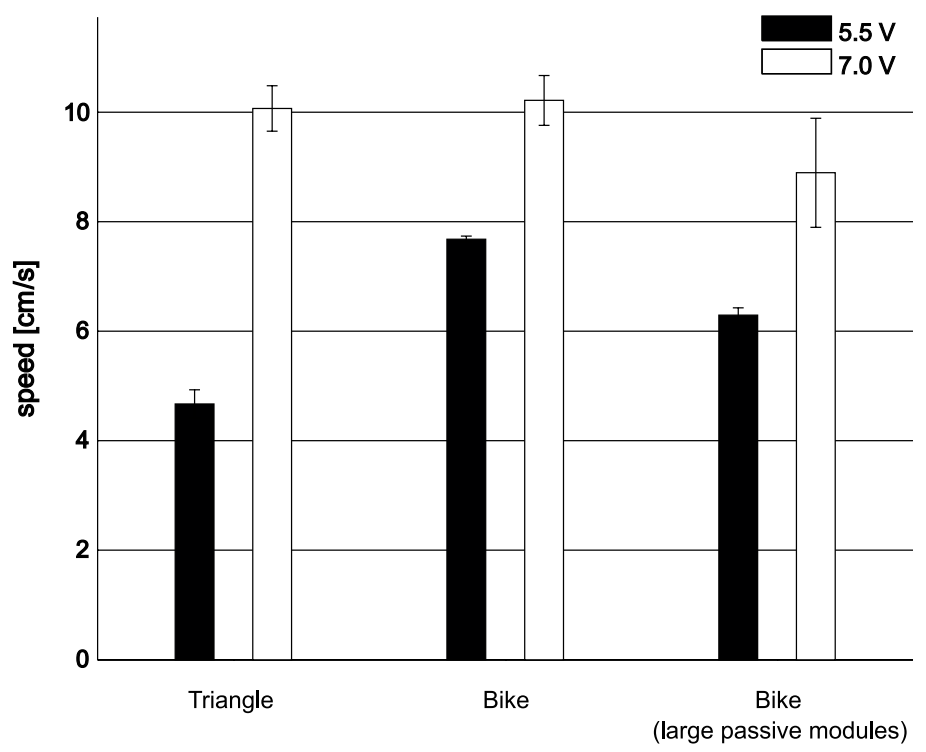

b

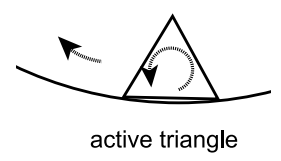

C

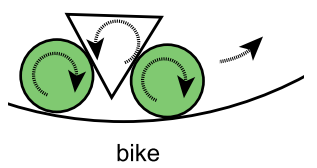

d

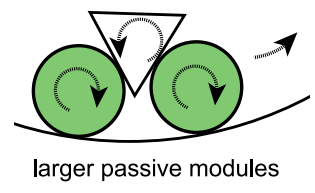

Figure 10. (a) Speed of wall-following behavior with different configurations of modules, tested under 5.5-V and 7.0-V power supply $(n=5)$. (b-d) The three configurations presented in (a).

been systematically investigated in experiments, but will be discussed based on our observations. The container size may influence the assembly time and the capillary effect. Given that the motion of an active module can be regarded as a quasi-random walk and obeys a diffusion law, the required time to travel a distance $L$ is proportional to $L^{2}$. This means that the assembly time is expected to increase with the size of the container. The size-or more precisely the curvature (shape) —of the container affects the contact surface area between a module and the container. As we described, the capillary attraction between a module and the container wall enabled reliable wall-following behavior; therefore, the lack of this force will most likely disable wall-following behavior and increase the chance of rotational motion. The applied voltage is a significant factor in the system's behavior. First, if the applied voltage is too high (i.e., the agitation level of an active module is too high to maintain a connection to a passive module), the system cannot aggregate [20]. Conversely, application of too low a voltage will cause a slow translational motion of an active module, and lead to longer assembly time. The influence of other parameters, such as viscosity, temperature, or the conductivity of the water, would require an extended study because they are interdependent and there are many practical restrictions. The water viscosity can be continuously altered by temperature to some degree, but that also affects the conductivity of the water. The prediction of behavior becomes difficult if these conditions are significantly changed. Some predictable changes occur, such as a decrease in translational motion of an active module with constant viscosity, as indicated in Equation 10, if the applied change in voltage is small. Unless the capillary force is affected, we suppose that continued observation of wall-following behavior is to be expected.

\subsection{Possibility of Different Combinations of Modules}

Scalability with respect to the number of modules is an important factor to be taken into account when designing self-organizing systems. However, the prediction is not trivial even when adding just one more module to the system. To examine this point, we performed the experiment with different sets of modules: two active and two passive module sets, and two active and three passive module sets. The most recognizable pattern was a group configuration consisting of two active and two passive modules forming a lattice structure with modules aligned alternately, but showing no noteworthy 
behavior. To attain efficient and selective assembly, modules should intrinsically feature hierarchical functions acting logically on the input; for example, a module prohibits further magnetic interaction on establishing an ideal connection. To date, only a few studies have investigated mechanically attained internal states that enable logical reactions $[15,26,29]$.

Related to this point, Figure 8 provides a unique insight into how simple modules could attain computation. It shows that the maximum number of passive modules that can connect to an active module is three (no matter how many passive modules exist). This is determined by the combination of morphologies of an active module and a passive module, where an increment of the number of connected passive modules causes a reduction of the attractive region. Such an alteration of the attractive region might also be done with internal states as introduced in [29]. In this way, morphology can be defined through an internal state and the module's outline.

\subsection{Design of the Shape and Function of the Self-Organization System}

Unlike proposals for static self-assembly systems, where the targets are mostly structures, proposals for dynamic self-assembly, self-organizing systems, and self-assembly robotics often aim at: (1) motion, which can be either positional motion (such as translational or rotational), or morphological motion (such as elongation or bending), and (2) function, especially function that is distinct from motion, such as metabolism or self-reproduction, and whose notions are often taken from biology. Designing a structure entails many features, and thus requires attention when designing. For example, the targeted attribute to control can be the length, size, stiffness, or topology. The relation between the autonomy of a module and the capability of the system is crucial for attaining a motion or function. In this regard, the level of a system's heterogeneity is a crucial variable to be considered when designing a self-organization system. The model presented here consists of two different-shaped modules. The wall-following motion presented here is a good example that shows when appropriate types of modules match, they exhibit advanced behavior even though they are simple modules. The relation between heterogeneity and autonomy is often a tradeoff, and an optimum needs to be derived. In the unusual case where the desired motion or function is already clear, (the library of such patterns has largely been assembled), an evolutionary optimization method may suit-for example, if the criterion is the swimming speed [30] or locomotion speed $[14,18]$. However, the difficulty here is always how to objectively find and set a good fitness function.

\section{Conclusion}

This article reports a self-organizing translational motion that is performed by a set of water-floating self-assembly modules. The system features a minimal hardware implementation for the operationnamely, one motor but no sensors - and shows reliable assembly and a stable wheeling behavior. During the experimental run, a floating module with a vibration motor (active module) roves around the area, and eventually picks up passive modules through magnetic attraction. By retaining contacts with multiple passive modules, an active triangular module transmits torque to the passive modules, inducing rotation of the passive modules, and consequently utilizes them as wheels. We analyze the entire self-organizing behavior as follows: (1) self-assembly phase, (2) rotational motion phase, and (3) wall-following phase. We also carefully investigated the associated characteristics. We further studied the influence of morphology, which, in this research, means the size of passive modules that act as wheels. This study provides insight into how morphology in assembled structures augments the aggregated functionalities of individual components of the structure.

\section{Acknowledgments}

Most of the topics discussed in Section 5 were prompted by the reviewers' suggestions. We sincerely thank the reviewers for these inputs. This work was partially supported by a Swiss National Science Foundation Fellowship PBZHP2-133472. 


\section{References}

1. Bishop, J., Burden, S., Klavins, E., Kreisberg, R., Malone, W., Napp, N., \& Nguyen, T. (2005). Programmable parts: A demonstration of the grammatical approach to self-organization. In IEEE/RSJ International Conference on Intelligent Robots and Systems (IROS) (pp. 3684-3691).

2. Boncheva, M., Andreev, S. A., Mahadevan, L., Winkleman, A., Reichman, D. R., Prentiss, M. G., Whitesides, S., \& Whitesides, G. M. (2005). Magnetic self-assembly of three-dimensional surfaces from planar sheets. Proceedinos of the National Academv of Sciences of the U.S.A., 102(11), 3924-3929.

3. Boncheva, M., Ferrigno, R., Bruzewicz, D. A., \& Whitesides, G. M. (2003). Plasticity in self-assembly: Templating generates functionally different circuits from a single precursor. Angewandte Chemie International Edition, 42, 3368-3371.

4. Bowden, N., Terfort, A., Carbeck, J., \& Whitesides, G. M. (1997). Self-assembly of mesoscale objects into ordered two-dimensional arrays. Science, $276(5310), 233-235$.

5. Breivik, J. (2001). Self-organization of template-replicating polymers and the spontaneous rise of genetic information. Entropy, 3, 273-279.

6. Cohn, M. B., \& Kim, C.-J. (1991). Self-assembling electrical networks: An application of micromachining technology. In International Conference on Solid-State Sensors and Actuators (pp. 490-493).

7. Fukuda, T., \& Kawauch, Y. (1990). Cellular robotic system (CEBOT) as one of the realizations of selforganizing intelligent universal manipulator. In IEEE International Conference on Robotics and Automation (ICRA) (pp. 662-667).

8. Gilpin, K., Knaian, A., \& Rus, D. (2010). Robot pebbles: One centimeter module for programmable matter through self-disassembly. In IEEE International Conference on Robotics and Automation (ICRA) (pp. 2485-2492).

9. Gracias, D. H., Tien, J., Breen, T. L., Hsu, C., \& Whitesides, G. M. (2000). Forming electrical networks in three dimensions by self-assembly. Science, 289(5482), 1170-1172.

10. Griffith, S., Goldwater, D., \& Jacobson, J. (2005). Robotics: Self-replication from random parts. Nature, 437, 636.

11. Grzybowski, B. A., Radkowski, M., Campbell, C. J., Lee, J. N., \& Whitesides, G. M. (2004). Self-assembling fluidic machines. Applied Physics Letters, 84, 1798-1800.

12. Grzybowski, B. A., Stone, H. A., \& Whitesides, G. M. (2000). Dynamic self-assembly of magnetized, millimetre-sized objects rotating at a liquid-air interface. Nature, 405, 1033.

13. Hauser, H., Ijspeert, A. J., Füchslin, R. M., Pfeifer, R., \& Maass, W. (2011). Towards a theoretical foundation for morphological computation with compliant bodies. Biological Cybernetics, 105, 355-370.

14. Hiller, J., \& Lipson, H. (2012). Automatic design and manufacture of soft robots. IEEE Transactions on Robotics, 28, 457-466.

15. Hosokawa, K., Shimoyama, I., \& Miura, H. (1994). Dynamics of self-assembling systems: Analogy with chemical kinetics. Artificial Life, 1(4), 413-427.

16. Ishiguro, A., Shimizu, M., \& Kawakatsu, T. (2006). A modular robot that exhibits amoebic locomotion. Robotics and Autonomous Systems, 54, 641-650.

17. Klavins, E. (2007). Programmable self-assembly. IEEE Control System Marasine, 27, 43-56.

18. Lipson, H., \& Pollack, J. B. (2000). Automatic design and manufacture of artificial lifeforms. Nature, 406, 974-978.

19. Mao, C., Thalladi, V. R., Wolfe, D. B., Whitesides, S., \& Whitesides, G. M. (2002). Dissections: Self-assembled aggregates that spontaneously reconfigure their structures when their environment changes. Iournal of the American Chemical Society, 124(49), 14508-14509.

20. Miyashita, S., Göldi, M., \& Pfeifer, R. (2011). How reverse reactions influence the yield rate of stochastic self-assembly. International Journal of Robotics Research, 30, 627-641.

21. Murata, S., Kurokawa, H., \& Kokaji, S. (1994). Self-assembling machine. In IEEE International Conference on Robotics and Automation (ICRA) (pp. 441-448).

22. Nakajima, K., Ngouabeu, A. M. T., Miyashita, S., Göldi, M., Füchslin, R. M., \& Pfeifer, R. (2012). Morphology-induced collective behaviors: Dynamic pattern formation in water floating elements. PLoS ONE, 7(6), e37805. 
23. Nakano, K., Uchihashi, S., Umemoto, N., \& Nakagama, H. (1994). An approach to evolutional system. In First IEEE Conference on Evolutionary Computation (CEC) (pp. 781-786).

24. Neubert, J., Cantwell, A. P., Constantin, S., Kalontarov, M., Erickson, D., \& Lipson, H. (2010). A robotic module for stochastic fluidic assembly of 3D self-reconfiguring structures. In IEEE International Conference on Robotics and Automation (ICRA) (pp. 2479-2484).

25. Oetomo, D., Daney, D., Harada, K., Merlet, J.-P., Menciassi, A., \& Dario, P. (2009). Topology design of surgical reconfigurable robots by interval analysis. In IEEE International Conference on Robotics and Automation (ICRA) (pp. 3085-3090).

26. Penrose, L. S. (1959). Self-reproducing machines. Scientific American, 200(6), 105-114.

27. Pfeifer, R., \& Bongard, J. C. (2007). How the body shapes the way we think. Cambridge, MA: MIT Press.

28. Pfeifer, R., Iida, F., \& Gomez, G. (2006). Morphological computation for adaptive behavior and cognition. International Congress Series, 1291, 22-29.

29. Saitou, K., \& Jakiela, M. J. (1995). Automated optimal design of mechanical conformational switches. Artificial Life, 2(2), 129-156.

30. Sims, K. (1994). Evolving 3D morphology and behavior by competition. In Artificial Life IV (pp. 28-39).

31. Stambaugh, J., Lathrop, D. P., Ott, E., \& Losert, W. (2003). Pattern formation in a monolayer of magnetic spheres. Physical Review E, 68, 026207-1-026207-5.

32. Michael, T., \& Lipson, H. (2010). Fluidic manipulation for scalable stochastic 3D assembly of modular robots. In IEEE International Conference on Robotics and Automation (ICRA) (pp. 2473-2478).

33. Tsutsumi, D., \& Murata, S. (2007). Multistate part for mesoscale self-assembly. In Proceedings of the SICE Annual Conference, (pp. 890-895).

34. White, P., Kopanski, K., \& Lipson, H. (2004). Stochastic self-reconfigurable cellular robotics. In IEEE International Conference on Robotics and Automation (ICRA) (pp. 2888-2893).

35. White, P., Zykov, V., Bongard, J., \& Lipson, H. (2005). Three dimensional stochastic reconfiguration of modular robots. In International Conference on Robotics Science and Systems (RSS) (pp. 161-168).

36. Wolfe, D. B., Snead, A., Mao, C., Bowden, N. B., \& Whitesides, G. M. (2003). Mesoscale self-assembly: Capillary interactions when positive and negative menisci have similar amplitudes. Lanomuir, 19(6), 2206-2214.

37. Yamaki, M., Higo, J., \& Nagayama, K. (1995). Size-dependent separation of colloidal particles in two-dimensional convective self-assembly. Lanomuir, 11(8), 2975-2978. 
